\title{
Liquid-Crystalline Polymer Gels VI. Preparation and Swelling Behavior of Cross-Linked Hydrogels of Poly(L-glutamic acid) Possessing Cholesteric Order
}

\author{
Yoshiki MatsuoKa, Ryoichi KISHI, and Masahiko Sisido* \\ Research Laboratory of Resources Utilization, Tokyo Institute of Technology, \\ 4259 Nagatsuta, Midori-ku, Yokohama 227, Japan
}

(Received November 30, 1992)

\begin{abstract}
Cross-linked gels of poly(L-glutamic acid) (PGA) possessing cholesteric liquidcrystalline order were prepared from the corresponding liquid-crystalline gels of poly $(\gamma$-benzyl L-glutamate). The PGA hydrogels were found to hold original liquid-crystalline order in the neutral $\mathrm{pH}$ range, but became isotropic at higher $\mathrm{pH}$ values. The phase change was reversible. The swelling with the increase of $\mathrm{pH}$ occurred through two stages. During the first stage that begins around $\mathrm{pH}=6$, a finger-print texture was observed under crossed polarizers. After the second stage of the swelling that begins around $\mathrm{pH}=9$, the finger-print texture was mostly disappeared. From a detailed study on the dissociation of PGA inside the gel, the first stage is attributed to a process of expansion of partially dissociated helical PGA chains and the second stage is the full expansion of fully dissociated PGA chains.

KEY WORDS Polypeptide / Hydrogel / Cholesteric Liquid Crystal / Swelling /
\end{abstract}

Polymer gels with a liquid-crystalline (LC) order (LC gels) have been reported recently from our ${ }^{1-5}$ and other laboratories. ${ }^{6-9}$ In our previous reports, ${ }^{1,2}$ lyotropic LC solutions of poly $(\gamma$-benzyl L-glutamate) (PBLG) have been crosslinked with appropriate diamino compounds to give LC gels keeping the original cholesteric or nematic LC order. The most striking property of the polypeptide LC gels was that they showed reversible interconversion between LC and isotropic phases with a change of solvent between helix-supporting one (dioxane) and breaking one (dichloroacetic acid). This indicates that the original LC order has been memorized in the cross-linked networks even after the gel was swollen to show an isotropic phase. Mechanical and optical properties of the gel were also changed reversibly with solvents. In the PBLG gels cross-linked under magnetic field, the reversible phase change induced an anisotropic change in the gel shape with higher expansion ratio perpendicular to the helix axis of PBLG. ${ }^{2,5}$

Although the anisotropic behavior of LC gels is interesting from a viewpoint as anisotropic chemomechanical or optical ${ }^{3,4}$ materials, the use of the PBLG LC gels is actually very limited because of volatile organic solvents, such as dioxane, dimethylformamide (DMF), and dichloroacetic acid (DCA), needed for their swelling. Therefore, preparation of LC hydrogels is an important step for practical application of LC gels.

For the preparation of LC hydrogels, two approaches may be possible. First, crosslinking of aqueous lyotropic LC's of watersoluble polymers may produce LC hydrogels. We have tried to cross-link poly(L-glutamic acid) (PGA) in concentrated aqueous solution. However, since the LC phase of aqueous PGA

* Correspondence should be addressed to this author. Present address: Department of Bioengineering Science, Okayama University, 3-1-1 Tsushimanaka, Okayama 700, Japan. 
solution is stable only at very high concentrations (about $45 \mathrm{wt} \%$ at room temperature) ${ }^{10}$ and it appears only after a very long period of standing, the cross-linking in a mixture of PGA with appropriate cross-linkers started before a full cholesteric structure was developed. Suto and Tashiro $^{6}$ cross-linked a concentrated aqueous solution of hydroxypropylcellulose with formaldehyde as cross-linker, and obtained a film that shows anisotropic behavior. Tanaka et al. ${ }^{7}$ prepared a film of PGA by cross-linking a concentrated solution of PGA with polyether diisocyanate. However, these films do not seem to possess full cholesteric LC structure and show no gel-like property.

The second approach to prepare LC hydrogels is to convert LC organogels to LC hydrogels. Since, as described above, the network of LC gels of PBLG memorizes the LC structure even under helix-breaking conditions, deprotection of benzyl ester groups of PBLG under strong acidic conditions may not destroy the LC structure of the gel. Therefore, a promising approach to prepare LC hydrogels is to prepare LC organogels of PBLG first, then remove benzyl groups under acidic conditions, and finally recover the LC structure in neutral solutions. In this paper, preparation and swelling behavior of the LC hydrogels of PGA are described. A preliminary report on the anisotropic properties of the PGA gel has been reported. ${ }^{5}$

\section{EXPERIMENTAL}

\section{Cholesteric Liquid-Crystalline Gel of PBLG in Dioxane}

Polymer gels with cholesteric liquid-crystalline order were prepared by cross-linking a $25 \mathrm{wt} \%$ solution of PBLG $\left(\mathrm{MW}=1.7 \times 10^{5}\right)$ in dioxane using triethylenetetramine as a crosslinker. ${ }^{1}$ The LC solution was placed in a thin cell of $200 \mu \mathrm{m} \times 1 \mathrm{~cm} \times 1 \mathrm{~cm}$ and stored at $25^{\circ} \mathrm{C}$ for 7 days to develop a full cholesteric structure. The cross-linking was carried out at $70^{\circ} \mathrm{C}$ for 7 days. After the reaction was com- pleted, the LC gel was peeled off from the cell to obtain a gel membrane. Three samples with different contents of the cross-linker $(9.0,4.1$, and $2.8 \mathrm{~mol} \%)$ were obtained. The content of the cross-linker was determined from elemental analysis after rinsing the gel with DMF and drying under vacuum. In the following experiment, attention will be focused on the gel with $9.0 \mathrm{~mol} \%$ of crosslinkers, unless otherwise mentioned.

\section{Cholesteric Liquid-Crystalline Hydrogel of $P G A$}

The above membrane was immersed in acetic acid containing $25 \mathrm{wt} \%$ of $\mathrm{HBr}$ at room temperature for $4 \mathrm{~h}$. The membrane was then rinsed with distilled water and with DMF. IR spectrum of dried sample in $\mathrm{KBr}$ disk showed that absorbance of benzyl group at 735 and $697 \mathrm{~cm}^{-1}$ disappeared after the acid treatment. Elemental analysis also indicated complete conversion of PBLG to PGA.

\section{Swelling Experiments}

Swelling experiments were carried out in organic solvents as well as in aqueous solutions at different $\mathrm{pH}$ values and ionic strengths. Acetate buffer $(\mathrm{pH}=3.7-5.7)$, phosphate buffer (6.2-7.0), barbital buffer (7.0-9.0), and borate buffer $(9.0-10.2)$ were employed. The buffer solutions of different ionic strengths were obtained by adding appropriate amount of $\mathrm{NaCl}$ to $I=0.01$ and 0.2 . The PGA gel in the $-\mathrm{COOH}$ form was dried and weighed to determine net weight $W_{0}$. It was swollen in $0.01 \mathrm{M} \mathrm{NaHCO}_{3}$ solution for 10 days and then shrunken in acetone. The latter was then immersed in the above buffer for one week at $25.5^{\circ} \mathrm{C}$ and weighed to determine equilibrium weight $W$. The degree of swelling was calculated as a ratio $W / W_{0}$.

\section{Measurements}

The textures of the LC gels were observed on an Olympus polarizing microscope under crossed polarizers. Absorption spectra were 
recorded on a Hitachi 320 spectrometer.

\section{RESULTS AND DISCUSSION}

Observation of Cholesteric Texture of PGA Gels in Organic and Aqueous Solvents

Figure 1 shows polarized microscope pictures of the PGA gels equilibrated in helix-

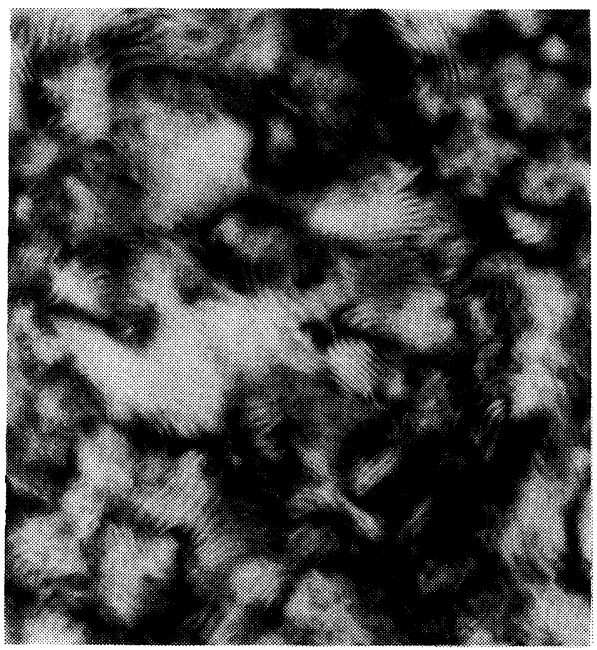

\section{(a) DMF}

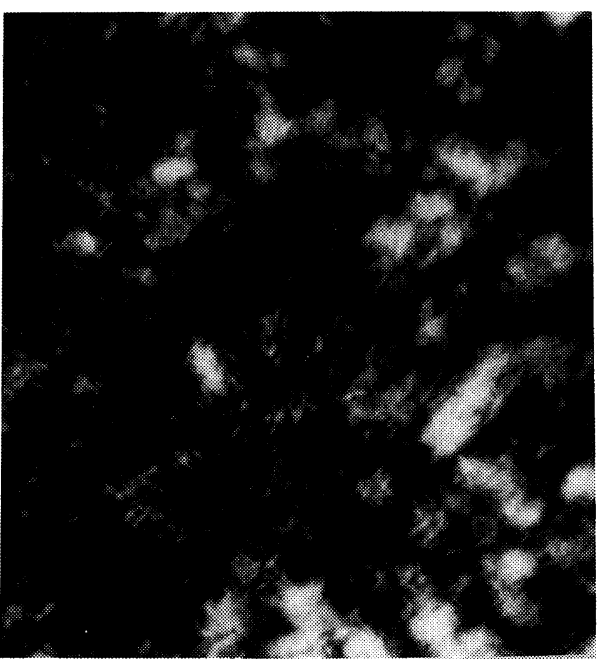

(c) DCA supporting solvents (DMF and dimethylsulfoxide (DMSO)), in helix-breaking solvent (DCA), and in poor solvent (dioxane). In the helix solvents, bright pictures were obtained under crossed polarizers, indicating an anisotropic structure of the gel. The pictures exhibit finger-print textures with about the same cholesteric pitch as the original PBLG LC gel

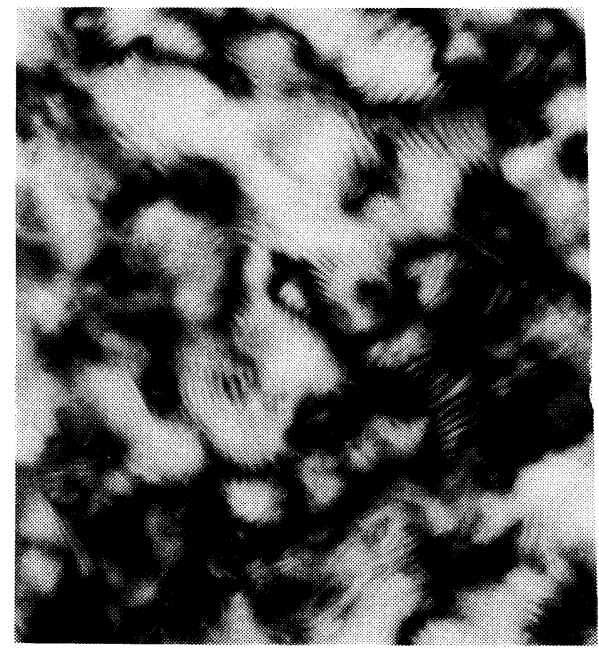

(b) DMSO

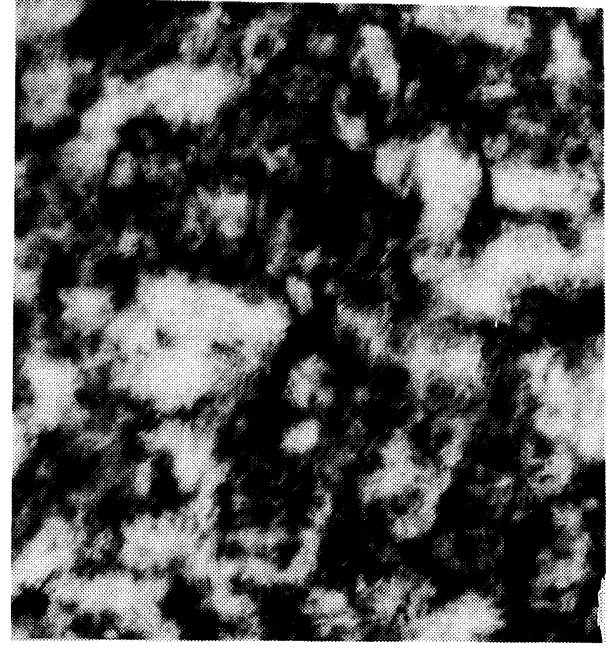

(d) DOX

Figure 1. Textures of PGA LC gels swollen in organic solvents. (a) in dimethylformamide, (b) in dimethylsulfoxide, (C) in dichloroacetic acid, and (d) in dioxane. 

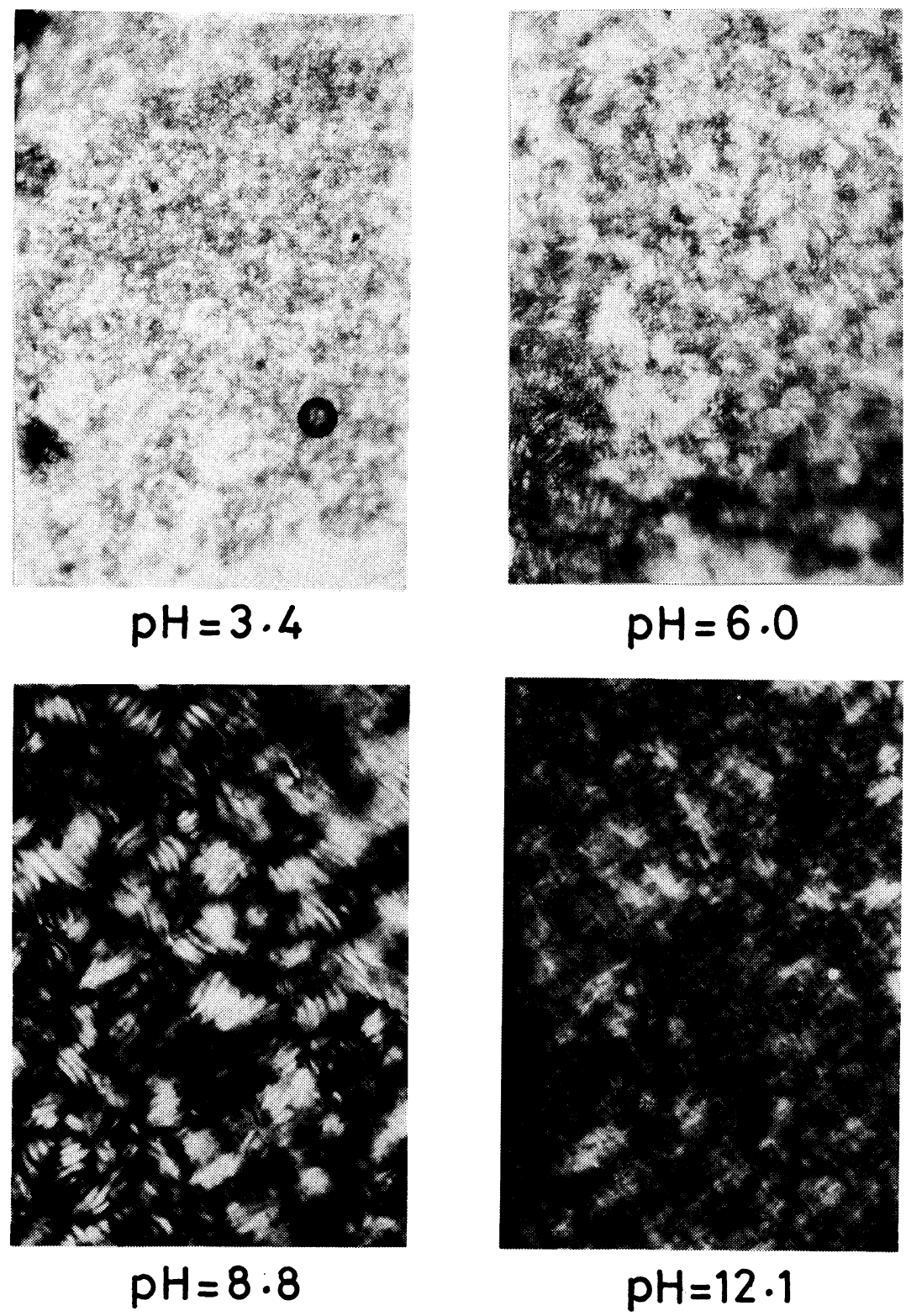

Figure 2. Textures of PGA LC hydrogels swollen at various $\mathrm{pH}$ values (ionic strength $=0.2$ ).

in dioxane. This observation indicates that the original LC structure of PBLG LC gel remained in the deprotected PGA gel. In DCA, the PGA film became isotropic and only very small light intensity was observed under crossed polarizers. However, when the picture was taken with long exposure time, a certain macroscopic structure can be observed (Figure 1c). In a poor solvent (dioxane), the PGA gel became shrunken. However, the anisotropy was more marked in the shrunken gel, resulting in a very bright picture. The gel structure in one solvent could be converted to the other structure in another solvent reversibly. For 
example, when an isotropic gel in DCA was immersed in DMF or in DMSO, the original finger-print texture was recovered after a few ten minutes.

\section{Swelling Behavior of PGA Hydrogel at Different pH Ranges}

Similar reversible change was observed when the PGA gel was immersed in water with different $\mathrm{pH}$ values. Figure 2 shows the texture observed at different $\mathrm{pH}$ values. Below $\mathrm{pH}=6$, the gel was shrunken and no clear finger-print texture was detected, but the anisotropy as indicated by the bright picture under crossed polarizers was very high. Above $\mathrm{pH}=6$, the gel became swollen and the finger-print texture appeared. At higher $\mathrm{pH}$ values, the gel expanded significantly and the texture was almost disappeared. The intensity of transmitted light diminished. Again, the $\mathrm{pH}$ dependent behavior was reversible.

The swelling ratios $\left(W / W_{0}\right)$ against $\mathrm{pH}$ values at different ionic strengths are shown in Figure 3. Interestingly, the swelling proceeds through two stages. The first stage begins at about $\mathrm{pH}=5$ and become sluggish above $\mathrm{pH}=7$. The second stage starts around $\mathrm{pH}=9$.

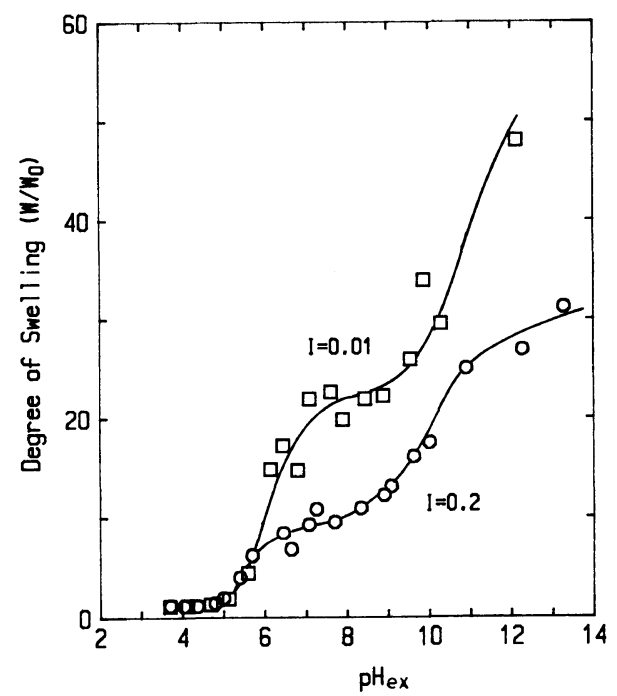

Figure 3. pH Dependence of swelling ratios of PGA LC hydrogels at two different ionic strengths.
The swelling is more significant at low ionic strenths, as expected from stronger electrostatic repulsions between anionic charges inside the gel. The two-step transition was observed at both ionic strengths but sharper at the lower ionic strength.

A likely reason for the observation of twostage swelling in the polypeptide hydrogel is a helix-coil transition associated with ionic dissociation of side chains. The helix-coil transition of free PGA molecules in solution has been reporred to occur at $\mathrm{pH}=5.0(I=0.2)$ and $\mathrm{pH}=5.8(I=0.01) .{ }^{11}$ The transition $\mathrm{pH}$ values are apparently in accordance with the $\mathrm{pH}$ values where the first stage of swelling starts to occur. This view should be altered, however, from a detailed study as described below.

\section{pH Values Inside the PGA Gel}

$\mathrm{pH}$ Values inside the gel was measured from absorption spectra of various $\mathrm{pH}$ indicators doped in the gel. The $\mathrm{pH}$ indicators employed at various $\mathrm{pH}$ ranges are: bromocresol green (3.8-5.4), bromocresol purple $(2.0-6.8)$, phenol red (6.8-8.4), thymol blue (1.2-2.8 and $8.0-9.6$ ). These indicators were dissolved

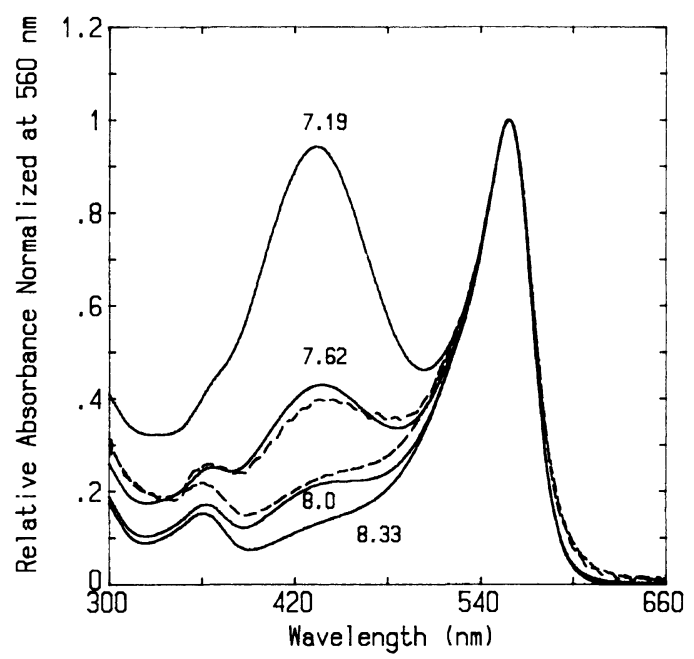

Figure 4. Absorption spectra of phenol red at various $\mathrm{pH}$ values (solid lines) and those of the dyes doped in PGA LC hydrogels (dashed lines). Spectra are normalized at $558 \mathrm{~nm}$. 
in buffers of known $\mathrm{pH}$ values and absorption spectra were measured. The indicators showed two interconvertible absorption peaks, with different relative intensities at different $\mathrm{pH}$ values. Calibration lines that relate the ratio of the peak intensities to the $\mathrm{pH}$ of solution were drawn and used to measure $\mathrm{pH}$ values inside the gel. Figure 4 exemplifies spectra of a $\mathrm{pH}$ indicator (phenol red) at different $\mathrm{pH}$ values (solid lines).

A film $(1 \mathrm{~cm} \times 1 \mathrm{~cm} \times 100 \mu \mathrm{m})$ of PGA gel was swollen in $0.01 \mathrm{M} \mathrm{NaHCO}$ solution and shrunken in acetone. The pretreated gel was swollen again in buffer solution $(I=0.2)$ of known $\mathrm{pH}$ containing about $10^{-4} \mathrm{M}$ of a $\mathrm{pH}$ indicator. The absorption spectrum of the dye-doped gel was measured and subtracted with that of a reference gel. Examples of absorption spectra of a gel doped with phenol red at two different $\mathrm{pH}$ values are shown in Figure 4 (dashed lines). From the absorption spectrum of $\mathrm{pH}$ indicators doped in the gel, the internal $\mathrm{pH}$ values were determined.

The internal $\mathrm{pH}$ values are plotted against the external $\mathrm{pH}$ values in Figure 5. It is evident that the internal $\mathrm{pH}$ is always lower than the external $\mathrm{pH}$ by about 0.3 . Qualitatively, the

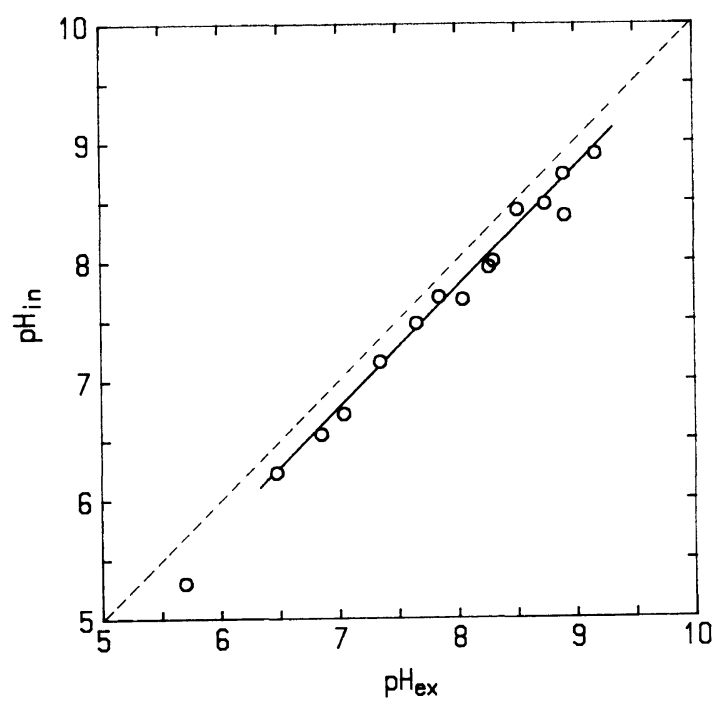

Figure 5. Relation between external $\mathrm{pH}$ values outside the gel and internal $\mathrm{pH}$ values inside the gel. lower internal $\mathrm{pH}$ values are explained in terms of the increased proton concentration due to highly dense carboxylate anions inside the gel. The difference between external and internal $\mathrm{pH}$ values is related to the difference of electrostatic potentials inside and outside the gel (Donnan potential), that is governed by the charge density of carboxylate anions. Therefore, the $\mathrm{pH}$ difference may be related to the degree of dissociation, as described quantitatively in the next section.

Relation between pH Difference and Degree of Dissociation

The $\mathrm{pH}$ difference $\left(\Delta \mathrm{pH}=\mathrm{pH}_{\mathrm{in}}-\mathrm{pH}_{\mathrm{ex}}\right)$ is determined by the Donnan potential, $\Delta \phi=$ $\phi_{\text {in }}-\phi_{\text {ex }}$, as,

$$
\Delta \phi=(2.303 R T / F) \Delta \mathrm{pH}
$$

where $F$ is the Faraday constant. The Donnan potential for membranes with fixed charges has been derived by Mauro ${ }^{12}$ and Hanai. ${ }^{13}$ Under conditions where only monovalent anions and cations are present, the Donnan potential is expressed by the ratio of concentration of fixed carboxylate anions inside the gel $C_{\mathrm{x}}$, and concentration of free anions (=cations) in the external solution, $C$.

$$
\begin{aligned}
\Delta \phi= & -(2.303 R T / F) \log \left[\left(C_{\mathrm{x}} / 2 C\right)\right. \\
& \left.+\sqrt{1+\left(C_{\mathrm{x}} / 2 C\right)^{2}}\right]
\end{aligned}
$$

Combining eq 1 and 2, we obtain a simple relation berween the effective molar concentration of carboxylate anion groups fixed in the gel and $\Delta \mathrm{pH}$.

$$
C_{\mathrm{x}}=C\left[\left(10^{-2 \Delta \mathrm{pH}}-1\right) / 10^{-\Delta \mathrm{pH}}\right] .
$$

Since $C_{\mathrm{x}}$ is a function of $\Delta \mathrm{pH}$, and $\Delta \mathrm{pH}$ is nearly constant over the $\mathrm{pH}$ range of 5.6-9.2 (Figure 5), it is concluded that the concentration of carboxylate anions is kept constant over the $\mathrm{pH}$ range studied. This indicates that the swelling proceeds with the dissociation of carboxyl groups keeping the effective concentration of anionic charges constant. 


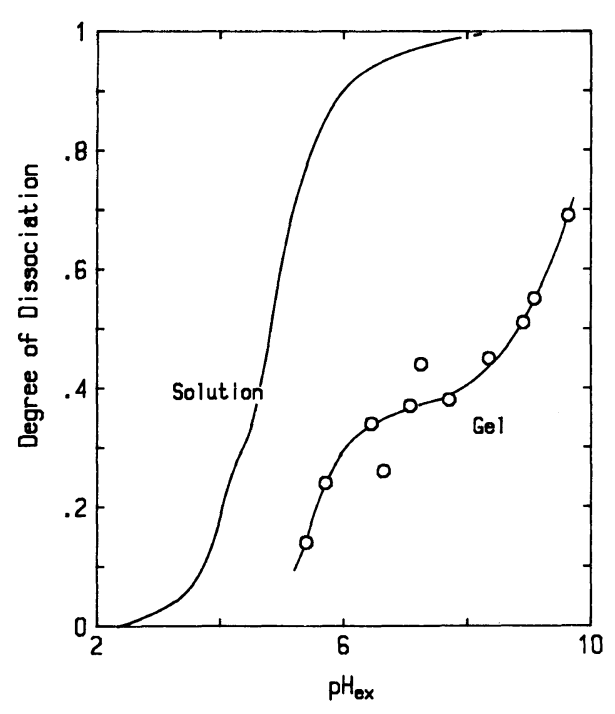

Figure 6. Dependence of degree of dissociation of carboxyl groups of PGA gel on external $\mathrm{pH}$ values. The solid line indicates the degree of dissociation of free PGA molecules in solution (data from ref 11). Ionic strength is 0.2 in both cases.

Total concentration of carboxyl groups in the gel $[\mathrm{COOH}]_{t}$, can be calculated from the swelling ratio $W / W_{0}$, taking the weight of crosslinkers into account. Then we can calculate the degree of dissociation $\alpha$.

$$
\alpha=C_{\mathbf{x}} /[\mathrm{COOH}]_{t}
$$

The degrees of dissociation at different external $\mathrm{pH}$ values are plotted in Figure 6. As qualitatively described above, the dissociation of carboxyl groups parallels the volume of the gel. In other words, the increase of anionic charges caused by the increase of external $\mathrm{pH}$ is compensated by the expansion of the volume of the gel, keeping the effective concentration of carboxylate anions inside the gel constant. The dissociation is markedly suppressed compared with that of free PGA molecules in aqueous solution (solid line). In aqueous solution the degree of dissociation reaches to unity at $\mathrm{pH}=8$ in $0.2 \mathrm{M} \mathrm{NaCl}$ solution, whereas it is about 0.4 at $\mathrm{pH}=8$ for $\mathrm{PGA}$ molecules inside the gel and did not reach to unity even near $\mathrm{pH}=10$.
Probable Mechanism of the Two-Stage Swelling

At this point we may propose a mechanism for the two-stage swelling of the PGA gel. The microscopic observation under crossed polarizers indicated that a cholesteric structure is present at $\mathrm{pH}=8.8$. Therefore, helical conformation is still dominant during the first stage of the swelling over the $\mathrm{pH}$ range of 5 to 8.8 . The first stage is therefore attributed to the swelling of partially dissociated $(\alpha=0.2-0.4)$ helical PGA molecules within a limiting volume $\left(W / W_{0}=c a .10\right)$ that is allowed for the networks originally formed on helical PBLG chains. At higher $\mathrm{pH}$ ranges, the helical conformation becomes unfolded due to the increased density of anionic charges and the LC structure begins to disappear. However, even at $\mathrm{pH}=12.1$, a certain macroscopic structure was remained (Figure 2), indicating that polypeptide chains incorporated in the networks cannot take fully random conformations even under helix-breaking conditions.

\section{FTIR Spectra of Freeze-Dried Samples}

In order to obtain information on the conforamtion of PGA molecules inside the gel, FTIR spectra were recorded on freeze-dried samples. Unfortunately, absorption of buffer components prevented the IR measurements under the same conditions as the swelling experiments. Therefore, the external $\mathrm{pH}$ was adjusted by a mixture of $\mathrm{HCl}$ and ammonia and no adjustment of ionic strength was made. This imposes some reservation on the interpretation of following results. FTIR spectra of PGA gel showed amide I and amide II peaks at 1654 and $1547-1550 \mathrm{~cm}^{-1}$ over the $\mathrm{pH}$ range of 2.2 to 10.6 , indicating that $\alpha$-helical conformation is predominant over the $\mathrm{pH}$ range. It is important to note that some portion of helical conformation still remains even at $\mathrm{pH}=11$. This is in accordance with the observation of some LC order in the PGA gel at $\mathrm{pH}=12$. Incidentally, a peak shift of amide II band from $1547 \mathrm{~cm}^{-1}$ to $1574 \mathrm{~cm}$ was 


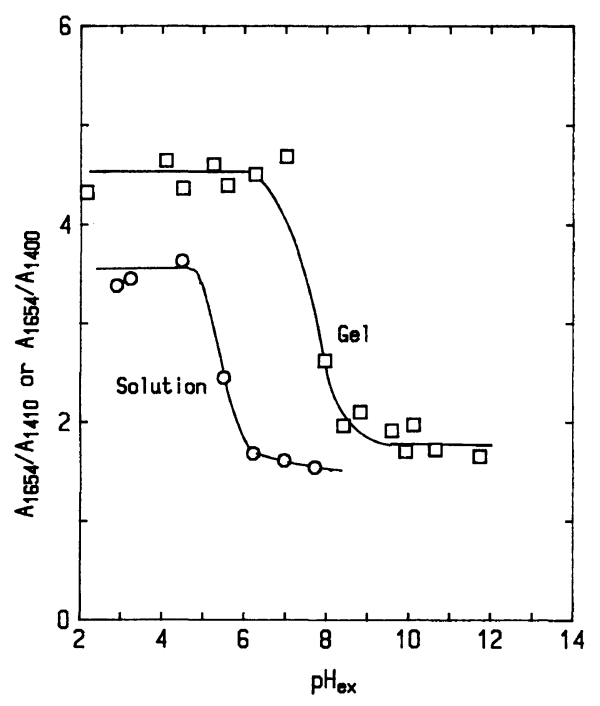

Figure 7. Ratios of infrared absorption at $1654 \mathrm{~cm}^{-1}$ (amide I) to that at 1410 or $1400 \mathrm{~cm}^{-1}$ (carboxylate anion) plotted against external $\mathrm{pH}$ values. The samples were freeze-dried from aqueous solution. The $\mathrm{pH}$ values of the latter was adjusted with hydrochloric acid and ammonia.

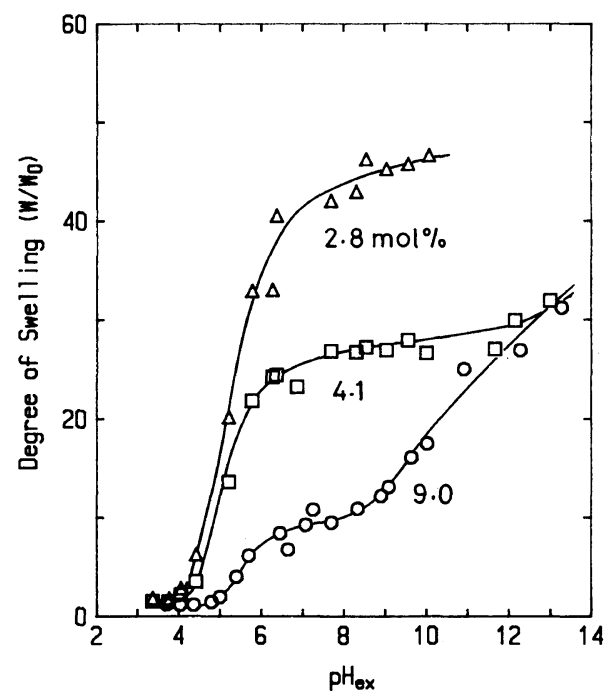

Figure 8. pH Dependence of swelling ratio of PGA LC hydrogels with different cross-linking densities. Ionic strength $=0.2$.

observed for freeze-dried sample of PGA in aqeuous solution, indicating helix-coil transition occurred at that $\mathrm{pH}$ in solution.

FTIR spectra also provide information on the degree of dissociation of carboxyl groups. Figure 7 shows the ratio of peak intensities at amide I band $\left(1654 \mathrm{~cm}^{-1}\right)$ to carboxylate band $\left(1400 \mathrm{~cm}^{-1}\right.$ for the gel or $1410 \mathrm{~cm}^{-1}$ for free PGA), plotted against the external $\mathrm{pH}$. It is clear that the ionic dissociation of the gel takes place at higher $\mathrm{pH}$ than that of free PGA in solution. These data are in accordance with the pictures drawn for the characteristics of the $\mathrm{pH}$-induced swelling of PGA gels, although a detailed comparison can not be made because of the different conditions.

\section{Effect of Cross-Linking Density on Swelling Profile}

The effect of cross-linking densities on the swelling behavior was examined and shown in Figure 8. No two-stage swelling was found when the cross-linking density was lower than $4.1 \mathrm{~mol} \%$. The gels with low cross-linking density show much highr swelling ratio than the highly cross-linked ones. Furthermore, the swelling starts at lower $\mathrm{pH}$ for gels with lower cross-linking density. These results are interpreted in terms of lesser restriction from cross-linked networks for the gels of lower cross-linking density. The two-stage swelling is a characteristics of PGA gels of highly dense cross-linking.

\section{CONCLUSIONS}

PGA hydrogels of cholesteric liquid-crystalline order was first prepared. They showed reversible change of liquid-crystalline phase to isotropic phase by the change of $\mathrm{pH}$ values. The swelling curves showed two stages for highly cross-linked PGA gels. During the first stage, the side-chain carboxyl groups were partially dissociated, but the helical conformation was maintained. In the second stage the dissociation became predominant and the helical conformation was largely unfolded. The mechanism of two-stage swelling is illustrated in Figure 9. Recently, the existence of multiphases has been reported for hydrogels of 


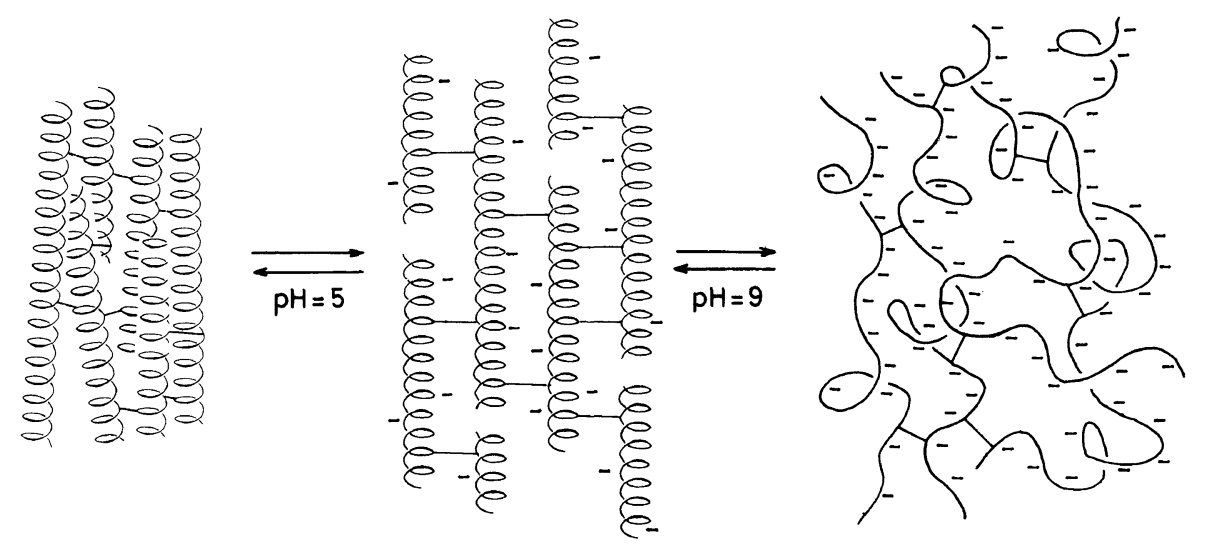

Figure 9. Schematic illustration of the two-step swelling of PGA LC hydrogels.

copolymers of anionic and cationic vinyl monomers. ${ }^{14}$ At this stage, it is not quite certain whether the present finding of the two-stage swelling indicates a general aspect of ionic hydrogels or a characteristic feature of polypeptide gels.

\section{REFERENCES}

1. R. Kishi, M. Sisido, and S. Tazuke, Macromolecules, 23, 3779 (1990).

2. R. Kishi, M. Sisido, and S. Tazuke, Macromolecules, 23, 3868 (1990).

3. M. Sisido and R. Kishi, Macromolecules, 24, 4110 (1991).

4. R. Kishi and M. Sisiso, Makromol. Chem., 192, 2723
(1991).

5. Y. Matsuoka, R. Kishi, and M. Sisido, Chem. Lett., 1855 (1992).

6. S. Suto and H. Tashiro, Polymer, 30, 2063 (1989).

7. T. Tanaka, T. Mori, K. Ogawa, and R. Tanaka, Polym. J., 11, 731 (1979).

8. a. R. Zentel, Liquid Crystals, 1, 589 (1986); b. R. Zentel, Angew. Chem. Int. Ed., 28, 1407 (1989).

9. F. Giasson, J. F. Revol, D. G. Gray, and J. St. Pierre, Macromolecules, 24, 1694 (1991).

10. E. Iizuka, Y. Kondo, and Y. Ukai, Polym. J., 9, 135 (1977).

11. A. Wada, Mol. Phys., 3, 409 (1960).

12. A. Mauro, Biophys. J., 2, 179 (1962).

13. T. Hanai, "Membranes and Ions" (In Japanese), Kagaku-Dojin, Kyoto, 1978, pp 200-221.

14. M. Annaka and T. Tanaka, Nature, 355, 430 (1992). 